\title{
Bernhard Sigmund Schultze-Jena
}

Nicht selten erweckt die Nachricht von dem Ableben eines schon seit langer Zeit von seiner Amtstätigkeit zurückgetretenen Gelehrten einiges Erstaunen darüber, daß dieser bis vor kurzem noch unter den Lebenden geweilt habe. Ganz und gar nicht der Fall ist dies bei Bern-hard Sigmund Schultze, der am 17. April d. J. im hohen Greisenalter sanft verschieden ist. Denn ScJmltze hat bis in die allerletzte Zeit seine geistigen Kräfte bewahrt und immer wieder durch wissenschaftliche Veröffentlichungen den Fachgenossen Kunde von seiner Arbeit gegeben; ja das in der Woehe seines Todes erschienene Heft des Centralblatts für Gynäkologie (No. 15 vom 12. April d. J.) bringt noch einen Auf-satz von ihm (Zur Kenntnis von der Einspritzung in die Vene der Nabel-

Monatsschrift í. Geburtshülfe u. Gynäkologie. Bd. XL IX. Heft 6. 26

378 Bernhard Sigmund Schultze-Jena $\dagger$.

schnur bei Blutung infolge adhärenter Placenta)! Wahrlich ein er-staunliches Maß ron geistiger Frische. Von dieser Frische konnten wir uns persönlich überzeugen, als wir ihm zur Feier seines 90. Geburts-tages die Glückwünsche darbrachten. Wenn auch das Augenlicht in den letzten Jahren versagte und auch die seit langem bestehende Schwerhörigkeit behinderte, so war er doch im übrigen von einer körperlichen und geistigen Kraft, die Erstaunen erregte; den zahl-reichen Gratulanten erwiderte er straff aufgerichtet in geistvoller Rede und gab interessante Sehilderungen aus seinem arbeitsreichen Leben.

Mit Schultze ist eine der allerersten Größen unseres Faches dahin-gegangen; ja es ist wohl nicht zu viel gesagt, wenn wir ihn als den Be-gründer der modern $\theta n$ Gynäkologie bezeichnen. Seine Lehren sind so innig in den Bestand des heutigen gynäkologischen Wissens über-gegangen, sie sind den Fachgenossen so bekannt, daß keine zu aus-führliche Schilderung seiner Arbeiten an dieser Stelle notwendig ist. Es soil im wesentlichen außer einer kurzen Darstellung seines äußeren Lebensganges ein Überblick über sein geistiges Schaffen gegeben werden.

Schultze stammt aus einer Gelehrtenfamilie. Er wurde am 29. De-zember 1827 zu Freiburg i. B. geboren .als Sohn von Karl August Sigmund Schultze. Dieser war Professor und Direktor der anatomischen und physiologischen Anstalten zu Freiburg i. B. (1821-1831) und Greifswald (1831-1868). Es war daher natürlich, daß Schultze anfangs anatomischen Studien sich zuwandte; dazu kam, daß sein älterer Bruder, der bekannte Anatom Max Schultze, schon in jungen Jahren Hervorragendes leistete und befruchtend auf den jüngeren Bruder einwirkte. Schultze promovierte 1851 in Greifswald mit einer Dissertation ,,De adipis genesi pathologica” und habilitierte sich ebenda 1853 für Anatomie. Aber bereits 1855 siedelte er nach Berlin über, als Assistent von D. W. H. Busch, und habilitierte sich hier für Ge-burtshülfe. Auch hier waren es zunächst anatomische und embryo-logische Studien, die er betrieb; so entstand u. a. die Arbeit , über die Entstehung der Doppelmonstra'; (Mon. f. Geburtsk. u. Frauenkrankh. Bd. VII, S. 247); er wies nach, daß das gleichzeitige Vorhandensein von 2 Keimbläschen in einem Ei der Entstehung der Doppelmiß-bildungen zugrunde liege und daß die verschiedenen Lagerungen der beiden Keimbläschen die verschiedenen Formen der Duplizität zu-stande bringen. 
Bald aber wandte er sich mehr geburtshülflichen Fragen zu. Aus den Arbeiten der Berliner Zeit sei besonders erwähnt die über ,, Respi-rationsbewegungen des Kindes im Uterus durch Auskultation wahr-nehmbar" (Dtsch. Klinik, 1857, No. 28; Mon. f. Geb., Bd. 10, S. 141). Dieses Thema der Ursachen und der Behandlung des Scheintodes der Neugeborenen hat er dann später vielfach und fruchtbringend be-arbeitet.

Bereits im Jahre 1858 wurde er als Nachfolger von Eãuard Martin auf den Lehrstuhl der Gynäkologie nach Jena berufen. Hier hat er dann sein Lebenswerk geschaffen. Er blieb der Universität Jena treu und war bis zum Jahre 1903, also voile 45 Jahre, im Amt. Seitdem Bernhard Sigmund Schultze-Jena $\uparrow .379$

iebte er in Jena im Ruhestand, aber auch weiter, wie oben schon ge-sagfc, wissenschaftlich tätig. $-$

Aus der großen Zahl der geburtshülflichen Schriften sei hier nur auf die Iíauptwerke hingewiesen. Im Jahre 1860 erschien die Arbeit liber das Nabelbläschen, in der er nachwies, daß dieses ein konstantes Gebilde in der Nachgeburt des ausgetragenen Kindes sei; treffliche Abbildungen sind dieser, wie vielen anderen Werken Schultzes bei-gegeben; Squlpze war selbst ein ausgezeichneter Zeichner.

Ebenfalls 1860 erschien die erste Auflage des Lehrbuches der Hebammenkunst (Leipzig, Wühelm Engelmann). Dieses treff liche Werk wurde immer mehr vervollkommnet und erschien in immer neuen Auflagen, die 15. Auflage im Jahre 1914. Es hat weiteste Ver-breitung gefunden, ist in viele fremde Sprachen übersetzt und wohl eines der besten Hebammenlehrbücher. Nicht nur für den Unterricht der Hebammen hat es sich bewährt, auch der junge Mediziner kann es mit großem Nutzen als die erste Einführung in die Geburtshülfe verwenden.

Weite Verbreitung fanden die großen Wandtafeln zur Schwanger-schaft und Geburtskunde, erste Auflage 1865, zweite Auflage 1888. Sie sind mit großer Meisterschaft entworfen und ausgeführt; die 2. Auflage hat der Schreiber dieses zum Teil mit entstehen sehen; auf dem großen Zeichentisch im Direktorzimmer der Klinik lag oft lange eine Tafel, an der Schultze immer wieder, freie Minuten benutzend, zeichnete und besserte.

Ein anderes verbreitetes Hilfsmittel für den geburtshülflichen Unterricht, das allgemein bekannte Phantom für die geburtshülflichen Operationen, wurde 1864 publiziert. Es gibt wohl keine geburtshülf-liche Unterrichtsanstalt, in der nicht das SchuUzesche oder ein ihm nachgebildetes Phantom im Gebrauch ware.

Sorgfältige Untersuchungen über denWechsel der Lage und Stellung des Kindes in den letzten Wochen der Schwangerschaft erschienen 1868 (Leipzig, Wilhelm Engelmann).

Die Kenntnis vom Mechanismus der spontanen Ausscheidung der Nachgeburt hat [Schultze wesentlich gefördert; Schíultzesche $\tau$ Modus neben dem ,D «»»c $<\mathrm{r} ø 1 / 8 \mathrm{schen}$ (Dtsch. med. Woch., 1880, No. 51/52).

Die oben bereits erwähnten Fragen über die Asphyxie der Neu-geborenen fanden vielfache Bearbeitung; die Resultate sind in dem 1871 erschienenen Werk ,,Der Scheintod des Neugeborenen" niedergelegt. In hervorragend scharfer Beweisführung brachte er Klarheit in die komplizierten Vorgänge, die bei Störung der Placentarzirkulation ein-treten, in die Lehre von der Einwirkung des.Geburtsaktes auf das Kind, in die Lehre von der intrautermen Atmung. Die reifste Frucht dieser Studien war die Methode der Wiederbelebung tief $\varepsilon$ cheintot geborener Kinder durch künstliche Pespiration mittels Schwingen (zuerst ver-öffentlicht Jenaer Ztschr., 1865). Diese Methode hat manchen Wider-spruch erfahren; es wurde behauptet, die Luft dringe dabei nicht ge-nügend in die Lungen und es seien Verletzungen des $\mathrm{K}^{1} / 8 \mathrm{des}$ zu be-fürchten. Es 
hat sich gezeigt, auch durch experimentelle Prüfung, daß solche Vorwürfe nur erheben könne, wer die Methode nicht riehtig

$26^{*}$

380

Bernhard Signiund Schultze-Jeria $\uparrow$.

ausführt. Macht man die Schwingungen genau nach den Vorschriften - es kommt dabei auf wichtige Einzelheiten an - so wie sie SchuUze gelehrt hat, so sind die Resultate ausgezeichnet. Wer es, wie Sehreiber dieses, wiederholt erlebt hat, daß Kinder, die schon als tot beiseite gelegt waren, die aber doch noch ab und zu einen Herzschlag zeigten, durch die Schwingungen wieder zu vollem Leben gebracht werden können, der möchte die Methode nicht missen.

Wohl noch entscheidender als in der Geburtshülfe hat SchuUze in der Lehre von den Frauenkrankheiten auf die Entwicklung unserer Disziplin eingewirkt.

SchuUze hat die Grundlagen geschaffen, auf denen erst die wicken-schaftliche Gynäkologie aufgebaut werden konnte. Ihm verdanken wir das Wesentlichste in der Methodik der gynäkologischen Diagnose. Wenn auch die bimanuelle Palpation von Einzelnen schon im 18. Jahr-hundert und in der ersten Hälfte des 19. angewendet wurde, so war die Kunst doch fast verloren gegangen, und erst SchuMze hat sie (neben Anderen wie Hoist, Hegar) ausgebaut und zur allgemeinen Einführung gebracht. Seine erste diesbezügliche Veröffentlichung stammt aus dem Jahre 1864 (Über Palpation normaler Eierstöcke, Jenaer Ztechr.). Aber schon im Anfang seiner gynäkologischen Tätigkeit war ihm dies Verfahren geläufig; so berichtete er über die Entfernung von Abort-resten durch kombinierte Manipulation bereits im Jahre 1856 (Mon. f. Geb., Bd. 7, S. 20). Bis ins einzelste hat SchuUze die Methode ver-vollkommnet, besonders auch durch die ausgiebige Anwendung der Rektalpalpation bzw. die kombinierte Recto - Vaginal Abdominal-palpation. Es sei z. B. an die von ihm angegebene Methode erinnert, den Stiel großer Ovarientumoren kombiniert zu tasten durch gleich-zeitiges Anhebenlassen des Tumors von außen (Zbl. f. Gyn., 1879, No. 6 und 1880, No. 1), sowie an die Diagnose und Lösung peritonealer Adhäsionen (Ztschr. f. Geb. u. Gyn, Bd. 14, 1887).

Sehr praktisch haben sich die von, Sputtze nach genauen Messungen gezeichneten Beckenschemata zum Einzeichnen der gynäkologischen Befunde bewährt (Mon. f. Geb., 1868, Bd. 32, S. 456 und 1869, Bd. 34, S. 151). Als später diese Schemata als Gummistempel hergestellt wurden, haben sie weite Verbreitung gefunden. Macht man es sich zur Regel, wie ich es nach Schultzes Anleitung seit Beginn meiner Tätigkeit tue, jeden gynäkologischen Befund zu zeichnen, so unter-sucht man viel exakter; es gibt nichts Bequemeres, als die an beliebige Stelle, des Krankenjournals einzufügenden Stempelabdrücke, in die man mit wenigen Strichen den Befund einzeichnet; beim Wieder-kommen der Patientin orientiert ein einziger Blick; etwaige Ver-änderungen, z.B. Größenzunahme des Uterus (alles wird in ein Drittel natûrlicher Größe gezeichnet) lassen sich leicht erkennen und fixieren.

Die erste Frucht seiner genauen Palpationsdiagnosen war die Feststellung der normalen Lage des Uterus an der Lebenden. Früher hatte man die Lage an der Leiche (meist Retropositio) für die noimale gehalten und meinte, die Anteflexion sei etwas Pathologieches. SchuUze zeigte, daß diese die normale Lage $\varepsilon e i$, daß aber vor allem zur Definition dieser normalen Lage die freie Beweglichkeit je nach dem Füllungs-

Bernhard Signound Schultze-Jena †. 381

zustand der Nachbarorgane u\&w. gehöre. Vielen Widerstand fanden Schultzes Ansichten, den er unermüdlich mit scharfer Kritik bekämpfte. Noch im Jahre 1885 wurde die Richtigkeit seiner Anschauungen be-zweifelt; in dem Lehrbuch der topographisehen Anatomie von Henke ist das 
bekannte Bchultzesche Bild der bimanuellen Palpation des Uterus wiedergegeben, aber zwischen die tastenden Hände ist nicht der Uterus, sondern die Harnblase gezeich.net; Henke behauptete alien Ernstes, daß das, was wir Gynäkologen für den Uterus palpieren, die Harnblase sei! Dem Thema der Lageveränderungen hat Schultze seine Haupt-forschung gewidmet. Im Jahre 1872 erschien die Arbeit über Versionen und Flexionen, speziell über die mechanisehe Behandlung der Rück-wärtslagerung der Gebärmutter (Arch. f. Gyn, Bd. 4). Das diesbezüg-liche Hauptwerk ,,Die Pathologie und Therapie der Lageveränderungen des Uterus” erschien 1881 (Berlin, August Hirschwald, Übersetzungen ins Englische und Französische). Auf diesem Gebiet hat Schultze Hervorragendes geleistet; seine Anschauungen haben sich zum aller-größten Teil als richtig erwiesen und bildeten die Grundlage, auf der die weitere Forschung aufgebaut wurde. Speziell die Behandlung der Retroflexion durch bimanuelle Reposition und Fixierung durch Pessar stellte er auf festen Boden. Er gab verschiedene Pessarformen an, an-gepaßt für den einzelnen Fall formbar (Gummiringe mit weichem Kupferdraht. Jenaer Ztschr., Bd. 5, 1869; später Zelluloidringe, die in heißem Wasser biegsam gemacht, sich in jede Form bringen lassen. Verhandl. d. Dtsch. Ges. f. Gyn., Halle 1888, S. 170). Bewährt hat sich auch sein Verfahren der Diagnose und Lösung peritonealer Ad-häsionen des retroflektierten Uterus und der entsprechend verlagerten Ovarien (Ztschr. f. Geb. u. Gyn., 1887, Bd. 14).

Weiter ist es die Lehre von der chronischen Endometritis, die Schultze wesentlich gefordert hat. Er lehrte die Dilatation des Uterus durch Laminaria durch streng antiseptisches Verfahren gefahrlos zu gestalten (Cbl. f. Gyn., 1878, No. 7). Den Probetampon gab er an als Mittel zur Erkennung des Uteruskatarrhs (Cbl. f. Gyn., 1880, No. 17 und 1889, No. 1.9). Seine Behandlungsmethode durch Dilatation und methodische Spülungen hat gute Resultate erzielt. Ganz besonderen Wert legte Schultze auf die Diagnose der Ursachen atypischer Uterusblutungen, für die der übrige Befund keinen ge-nügenden Aufschluß gab, durch Dilatation (meist durch Laminaria) und Austastung des Uterus unter Entgegentasten von außen. Zweckentsprechende Instrumente gab er an, um kleine Polypen usw., die so gefunden wurden, zu entfernen (Löffelzangen, Illustr. Vierteljahrs-schr. d. ärztl. Polytechnik, 1880).

So sind es wesentlich Kapitel der sogenannten kleinen Gynäkologie, die gerade für den Praktiker besonders wichtig sind, die Schultze kulti-vierte. Aber auch am Ausbau der operativen Gynäkologie nahm er reichen Anteil. Schon 1871 berichtete er über von ihm ausgeführte Ovariotomien (Arch. f. Gyn., Bd. 2). Schultze gehörte zu den Wenigen, die die Richtigkeit der Lehren Semmelweis' schon zu dessen Lebzeiten erkannten und praktisch verwerteten. Die Errungenschaften der anti-

382 Bernhard Sigmund Schultze-Jera $\uparrow$.

septischen bzw. aseptischen Maßnahmen wurden auf der ScJwltzeschen Klinik in reichem Maße ausgenutzt. Schon sehr frühzeitig wurden bei Schiãtze die Instrumente ausgekocht, als noch keine besonderen Appa-rate dafür angegeben waren.

Von Einzelheiten operativer Arbeiten sei erwähnt Schiultzes Methode der operativen Heilung der urethralen Inkontinenz beim Weibe (Arch. f. Gyn. 1882, Bd. 20 u. Corr.-Bl. d. allg. ärztl. Ver. von Thür., 1888, No. 3), sowie seine Mitteilung über Heilung schwerer Puerperalerkrankung durch Amputation des septisch infizierten Uteruskörpers (Dtsch. med. Woch., 1886, No. 44); es war der erste diesbezügliche Fall; er ist mir persönlich in ganz besonderer Erinnerung, da ich ihn als Assistent zuerst in der Poliklinik, dann in der Klinik behandelte. Alle Versuche, die retinierte Placenta zu entfernen, schlugen fehl, der starre lange Cervikalkanal wurde nicht weiter; als septische Infektion eintrat, ergab sich die Indikation durch Laparotomie den Krankheitsherd zu entfernen, was mit bestem Erfolge gelang. - 
So hat Schultze eine überaus reiche wissenschaftliche Arbeit auf dem Gebiete der Geburtshülfe und Gynäkologie geleistet. Alle seine Arbeiten, auch die zahlreichen Einzelleistungen, die hier nicht erwähnt wurden, sind Musterbilder kritikvoller und prägnanter Darstellung. Gleichen Schritt mit seiner wissenschaftlichen Arbeit hielt seine praktische Tätigkeit als Arzt und Lehrer. Von weit her strömten ihm die Kranken zu und er hat in das damals noch sehr kleine Jena ein gynäkologisches Material gezogen, wie es zu der Zeit celbst auf großen UniVersitätsklihiken noch nicht vorhanden war.

Sein Unterricht war besonders fruchtbringend. Speziell die Aus-bildung in der praktischen Gynäkologie war musterhaft. Die Jenaer Klinik hatte ein großes klinisches Material von nicht operativen Fallen. Gerade diese waren für den Unterricht besonders wertvoll. In dem von der Klinik getrennt gehaltenen Untersuchungskurs wurden nicht nur Schwangere untersucht, wie das auf den meisten Kliniken der Fall war, sondern in ausgiebigster Weise wurde die gynäkologische

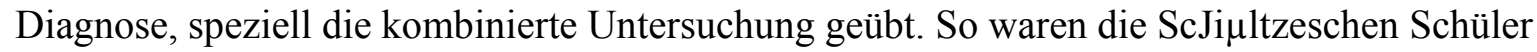
lange Zeit gerade gynäkologisch für die Praxis bescer gerüstet, als die Schüler mancher anderer, größerer Universitäten.

Auch rein menschlich war Schultze eine prächtige, wahrhaft vor-nehme und dabei liebenswürdige Persönlichkeit, die bei seinen Schülern und Freunden das Gefühl dankbarer Verehrung hervorrief. Trotz vieler hoher Titel und Auszeichnungen war er stets, wenn auch seines Werte $\beta$ bewußt, einfach im Wesen, nie den Überlegenen hervorkehrend. Dies zeigte sich ganz besonders darin, daß er auf jede sachliche Einwendung, auch des jüngsten Assistenten, liebevoll einging und stets bereit war, auch andere Meinung gelten zu lassen. Er erzog seine Assistenten zu größter Selbständigkeit und ließ ihnen die größte Freiheit. Ich selbst bin 13 1/2 Jahre sein Assistent gewe een, von 1884-1897; eine so lange Assistentenzeit ware nicht möglich gewesen ohne die große Liberalität, die mir Schultze zuteil werden ließ.

So lebt Schultze in der Erinnerung derer, die das Glück hatten, ihm persönlich nahe zu treten, fort als eine hervorragende Gestalt.

Bernhard Sigmund Schultze-Jena †. 383

In der Wissenschaft aber hat er dauernde Werte geschaffen; sie wird seinen Namen fur alle Zeiten unter die allergrößten ihres Reiches einreihen.

E. S. Sehultzes wissenschaftliehe Arbeiíen.

De adipis genesi pathologica. Diss.-Inaug. Greiíswald 1851. - Über anomale Duplizität der Axenorgane. Virchows Arch. 1855. Bd. 7. - Phlebitis umbilicalis, Entzündung und Vereiterung beider Submaxillar-und Sublingual-Speicheldrüsen. Mon. f. Geb. 1856. Bd. 7. S. 17. - Abortus eines 4 monatlichen Fötus, künstliche Lösung der Placenta am 12. Tage. Ebenda. S. 20. - Über die Entstehung der Doppelmonstra. Mon. f. Geb. 1856. Bd. 7. S. 247. -> Harnsaurer Niereninfarkt, intrauterine Leichen-starre. Dtsch. Klinik. 1857. No. 41. - Erwiderung an v. Bitgen (über Doppelmonstra). Mon. f. Geb. 1857. Bd. 9. S. 459. - Respirations-bewegungen des Kindes irn Uterus durch Auskultation wahrnehmbar. Dtsch. Klinik. 1857. No. 28; Mon. f. Geb. Bd. 10. S. 141. - Spina bifida und Hydrocephalus. Mon. f. Geb. Bd. 10. S. 5. - - Geburt und Sektion eines wasserköpfigen Kindes. Mon. f. Geb. 1858. Bd. 11. S. 105. -Cysten-förmige Auftreibung des rechten Ovarium durch Blutextravasate bei einem totgeborenen Kinde. Mon. f. Geb. Bd. 11. S. 170. - Zwillingsgeburt mit 5 vorliegenden Extremitäten. Mon. f. Geb. Bd. 11. S. 355. - Über die Indikation zur Kephalothrypsie. Allg. med. Centr.-Ztg. 1858. No. 50 und 51.$>$ Über Erhaltung und Zerreißung des Dammes bei der Geburt. Mon. f. Geb. 1858. Bd. 12. S. 241. - Wahrnehmung intrauteriner Atem-bewegung. Dtsch. Klinik. 1859. No. 3 und 10. Vesicula umbilicalis. Programm Jena 1860. - Lehrbuch der Hebammenkunst, 1 bis 15. Aufl.; 1. 
Aufl. 1860, 15. Aufl. 1914, Leipzig, W. Engelmann. - Ein Fall von Heterotaxie der Bauch- und Brusteingeweide. Virchows Arch. 1861. Bd. 22. -' Das Nabelbläschen, ein konstantes Gebilde in der Nachgeburt des ausgetragenen Kindes. Leipzig 1861. W. Engelmann. - Ein Kaiser-schnitt. Programm Jena 1862. -Eine ausgetragene Tubo-TJterinschwanger-schaft, Überwanderung des Eies. Würzburger med. Ztschr. 1863. Bd. 3. S. 178. - Exstirpation eines Gebärmutterfibroid. Jenaer Ztschr. 1864. - Über Palpation normaler Eierstöcke. Jenaer Ztschr. 1864. - > Eine Extrauterinschwangerschaft. Jenaer Ztschr. 1864. -> Geburtshülfliches Phantom. Jenaer Ztschr. 1864. - Zur Kenntnis der Todesart des Kindes bei vorzeitiger Lösung der Placenta. Jenaer Ztschr. 1864. - Über Super-fökundation und Superfötation. Jenaer Ztschr. 1865. Bd. 2.Wandtafeln zur Schwangerschaft und Geburtskunde. 20 Tafeln imp. folia. 1. Aufl. 1865, 2. Aufl. 1888. - Über die beste Methode der Wiederbelebung scheintot geborener Kinder. Jenaer Ztschr. 1865. Bd. 2. - Zur Kenntnis von der Einwirkung des Geburtsaktes auf die Frucht, namentlich in Beziehung auf Entstehung von Asphyxie und Apnoe des Neugeborenen. Virchows Arch. 1866. Bd. 37. - Die genetische Bedeutung der velamentalen Insertion des Nabelstranges. Virchows Arch. 1866. Bd. 37; 2. Mitteilung Jenaer Ztschr. 1867. Bd. 3.-Kindesmord oder nicht? Wien. Ztschr. f. gerichtl. Med. 1866. -Verminderung der im Becken gegebenen Widerstände. Jenatr Ztschr. 1867. Bd. 3. -Untersuchungen über den Wechsel der Lage und Stellung des Kindes in den letzten Wochen der Schwangerschaft. Leipzig 1868. W. Engelmann. - Die Placentarrespiration des Fötus. Jenaer 384

Bernhard Sigmund Schultze-Jena $\dagger$. Ztschr. 1868. Bd. 4. -John Mayow, Über Apnoe und Placentarrespiration. Jenaer Ztschr. 1868. Bd. 4. -Der Hermaphrodit Katharina Hohmarm aus Melrichstadt. Virchows Arch. Bd. 43. - Zur forensischen Diagnose des Geschlechts. Jenaer Ztschr. 1868. Bd. 4. - Über die narbenförmigen Streifen in der Haut des Oberschenkels. Jenaer Ztschr. 1868. Bd. 4. - Palpation und graphische Notierung. Naturforscherversammlung Dresden 1868; Mon. f. Geb. 1868. Bd. 32. S. 456; Mon. f. Geb. 1869. Bd. 34. S. 151; Jenaer Ztschr. 1869. Bd. 5. S. 113. - Eine Gebärmutter mit mindestens 50 Fibroiden. Jenaer Ztschr. 1869. Bd. 5. - Pessarien aus weichem Kupferdraht und vulkanisirtem Gummi. Jenaer Ztschr. 1869. Bd. 5. - Heckers Gesichtslagen. Arch. f. Gyn. 1870. Bd. 1. - Scheintod des Neugeborenen. 1871.-Vier Ovariotomien. Arch. f. Gyn. 1871. Bd. 2. Über Versionen und Flexionen, speziell über die mechanische Behandlung der Pvückwärtslagerungen der Gebärmutter. Arch. f. Gyn. 1872. Bd. 4. S. 373. -- Über Zwillingsschwangerschaft. Samml. klin. Vortr. 1872. No. 34. - Über die Lageveränderungen der Gebärmutter. Samml. klin. Vortr. 1873. No. 50. - Prophylaxe des Kindbettfiebers. Oorr.-Bl. d. allg. ärztl. Ver. v. Thür. 1873. No. 8/9. - Recension Fritsch. Jenaer Litt. Ztg. 1874. - Recension Winded. Jenaer Litt. Ztg. 1875. - Über die pathologische Anteflexion der Gebärmutter und die Parametritis posterior. Arch. f. Gyn. 1875. Bd. 8. - Zur Kenntnis von der Lage der Eingeweide. Arch, f. Gyn. 1876. Bd. 9 - Zur Frage vonderpathologischen Anteflexion der Gebärmutter. Arch. f. Gyn. 1876. Bd. 9. - Über Ovario-tomie. Dtsch. Ztschr. f. prakt. Med. (Kunze). 1876. Recension Winded. Jenaer Litt. Ztg. 1877. - Asphyxie, in Gerhards Handb. d. Kinderkrankh. 1877. Bd. 2. - Icterus neonatorum. Ebenda. - Die Erweifcerung des Uterus durch Laminaria digitata. Zbl. f. Gyn. 1878. No. 7. - Die exakte Ermittlung der Lage des Uterus in der lebenden Frau. Zbl. f. Gyn. 1878. No. 11. - Eine neue Methode der Reposition hartnäckiger Retroflexione П. Zbl. f. Gyn. 1879 No. 3. - Zur Diagnose großer Ovarientumoren. Zbl. f. Gyn. 
1879. No. 6. - Zur Klarstellung der Indikationen für Behandlung der Ante- und Retroversionen und -flexionen der Gebärmutter. Samml. klin. Vortr. 1879. No. 176. - Über Indikation und Methode der Dilatation des Uterus. Wien. med. B1. 1879. No. 42 /45. - Zur Diagnose großer Ovarientumoren II. Zbl. f. Gyn. 1880. No. 1. - Zur Kenntnis von den Methoden der Dilatation des Uterus. Zbl. f. Gyn. 1880. No. 15. - Zur Kenntnis von den Ursachen des Icterus neonatorum. Virchows Arch. 1880. Bd.81. -Der Probetampon, ein Mittel zur Erkenmmg der chronischen Endometritis. Zbl. f. Gyn. 1880. No. 17. -Zwei gynäkologische Preis-aufgaben. Wien. med. B1. 1880. No. 41/42. - Löffelzangen zur Entfernung kleinerer Neubildungen von der Innenwand des Uteruskörpers. Illustr. Vierteljahrsschr. d. ärztl. Polytechnik. 1. X. 1880. - Schemata. Jena. Gustav Fischer. - Über den Mechanismus der spontanen Ausscheidung der Nachgeburt und über den CVedéschen und den Dubliner Handgriff. Dtsch med. Woch. 1880. No. 51/52. - Die Pathologic und Therapie der Lageveränderungen des Uterus. Berlin 1881. August Hirschwald; Aus-zug daraus Wien. med. B1. 1881. No. 49. - Regeln für die Pflege der Wöchnerinnen, Taschenbuch für Krankenpfleger $1 / 8$ nen. Weimar 1882. 4. Jahrg. - Zur Dilatation des Uterus. Naturforschervers. Eisenach 1882. Arch. f. Gyn. Bd. 20. - Operative Heilung urethraler Inkontinenz beim

Bernhard Sigmund Sehultze-Jena †. 385

Weibe. Arch. f. Gyn. 1882. Bd. 20. - Über Ätiologie und Prophylaxe der Genitalerkrankungen des Weibes. Wien. med. B1. 1882. No. 52. - Icterus neonatorum (gegen Hofmeier). Fortschr. d. Med. 1883. No. 1. - Gynäkologisch $\theta$ Behandlung und Geistesstörung. Berl. klin. Woch, 1883. No. 23. - ÜbӨr Behandlung der Uterusblutungen. Holzers Taschenkalender f. Badeärzte. 1884. $>$ Unser Hebammenwes $\beta n$ und das Kindbettfieber. Samml. klin. Vortr. 1884. No. 247. -

Troicart-Schnäpper zur Punktion des Darmes. Illustr. Mon. d. ärztl. Polytechnik. 1884. - Über die Wieder-belebung tief scheintot geborener Kinder durch künstliche Respiration mittels Schwingen. Wien. med. B1. 1885. No. 1/2. - Anleitung zur Wendung auf den Fuß und zum Gebrauchder Geburtszange (für Hebammen). Leipzig 1885. W. Engelmann. - Zur Palpation der Beckenorgane. Zbl. f. Gyn. 1885. No. 43. - Über Totalexstirpation des karzinomatösen Uterus. Dtsch. Med.-Ztg. 1886. No. 2, 3, 4 und 16. -· Heilung schwerer Pueiperal-erkrankung durch Amputation des septisch infizierten Uteruskörpers. Naturforschervers. Berlin 1886; Dtsch. med. Woch. 1886. No. 44. - Über velamentale und placentale Insertion der Nabelschnur. Arch. f. Gyn. 1887, Bd. 30. - Über Diagnose und Lösung peritonealer Adhäsionen des retroflektierten Uterus und der entsprechend verlagerten Ovarien. Zeit-schrift f. Geb. u. Gyn. 1887. Bd. 14. - Referate über Gynäkologie in Fortschr. d. Med. 1883 u. ff. - Bericht über die in den Jahren 1884, 1885, 1886 ausgeführten Laparotomien. Corr,-Bl. d. allg. ärztl. Ver. v. Thür. 1887. No. 3. -Über operative Heilung der urethralen Inkoatinenz beim Weibe. Corr.-Bl. d. allg. ärztl. Ver. v. Thür. 1888. No. 3. - Über Pessarien. Verhandl.d. Dtsch. Ges. f. Gyn. Halle 1888. S. 170. Demonstration von Präparaten. Ebenda. S. 225. - Der Probetampon und sein Wert für Erkennung der chronischen Endometritis. Zbl. f. Gyn. 1889. No. 19; Dtsch. med. Woch. 1889. No. 21; Wien. med. B1. 1889. No. 20/21: Corr.-Bl. d. allg. ärzt. Ver. v. Thür. 1889. No. 5. -> Über die Anwendungs-weise und die Vorzüge der Zelluloidringe. Mannheimer Vereinsbl. 1889. -· Atembewegung und Herzaktion asphyktischer Früclite. Zbl. f. Gyn. 1890. No. 6. -Bildliche Darstellung des gynäkologischen Tastbefundes. 10. Intern. Kongreß Berlin 1890. Dtsch. med. Woch. 1890. No. 37. - Zur Therapie hartnäckiger Retroflexionen der Gebärmutter. Corr.-B1. d. allg. ärztl. Ver. v. Thür. 1891. No. 1/2; Samml. klin. Vortr., N. F. 1891. No. 24. - Reverdins Vorrichtung zur Erleichterung der Exstirpation großer Unter-leibstumoren. Zbl. f. Gyn. 1893. No. 6.-Zur Wiederbelebung tief scheintot geborener Kinder durch Schwingen. Zbl. f. Gyn. 1893. 
No. 15. - Über den Plan, den Besuch der psychiatrischen Klinik für die Studierenden der Medizin obligatorisch zu machen. Als Manuskript gedruckt. 1893. - Die Psychiatrie, Prüfungsgegenstand für alle Ärzte ? Jena 1893. G. Fischer. - · 1st Fraktur des Schlüsselbeins Kontraindikation ein tief asphyktisch geboreries Kind zu schwingen ? Zbl. f. Gyn. 1894. No. 8. Echinococcus des rechten Ovarium neben zahlreichen Echinokokken im Peritoneum. Festschr. z. F0ier des 50 jährigen Jubiläums der Ges. f. Geb. u. Gyn. in Berlin 1894. - Zur Ätiologie und Behandlung des Pruritus vulvae. Zbl. f. Gyn. 1894. No. 12. - Caput obstipum und Facialislähmung im Gefolge einer spontanen Geburt aus erster Schädellage. Zbl. f. Gyn. 1894. No. 39; Ztschr. f. Geb.u. Gyn. Bd.31. - Bericht über sogenaimte Spontanheilung einer mittelgroßen Blasen-Scheiden-Gebärmutterfistel, nebst prophy-

386 Bernhard Sigmund Schultze-Jena $\dagger$. laktischen Bemerkungen. Zbl. f. Gyn. 1895. No. 27.-Einiges über Pathologic und Therapie der Retroflexio uteri. Mon. f. Geb. u. Gyn. 1896. Bd. 3. S. 1 u. 101. - Mein Votum zur Revsion der medizinischen Prüfungen. Berlin 1896. Mittler u. Sohn. - Die Psychiatric, Prüfungsgegenstand für alle Ärzte ? 4. Aufsatz über das genannte Thema. Jena 1896. G. Fischer.- Vom Schwingen tief scheintot geborener Kinder. Ztschr f. prakt. Ärzte. 1896. No. 15. - Die Psychiatric Prüfungsgegenstand für alle Ärzte bei entsprechend verlängerter Studienzeit. $\delta$. Aufsatz über das genannte Thema. Jena 1896. G. Fischer. - Über die beim Scheintod Neugeborener vorliegenden Indikationen. Zbl. f. Gyn. 1896. No. 37. - Referat über Retro version und Retroflexion des Uterus. Verhandl. d. Dtsch. Ges. f. Gyn. Leipzig 1897. S. 50. -Demonstration eines Steinfötus. Ebenda. S. 186.- Demonstration von Reverdins Apparat. Ebenda. S. 408. - Das gebvirtshülfliche Phantom. Mon. f. Geb. u. Gyn. 1898. Bd. 7. S. 244. - Über Axendrehung des Uterus durch Geschwülste. Ztschr. f. Geb. \ı. Gyn. 1898. Bd.38. S. 157. - Vier Wandtafeln zur Diagnose und bimanuellen Reposition des retroflektierten Uterus. Leipzig 1898. W. Engelmann. Wieder ein Echinococcus ovarii dextri. Ztschr. f. Geb. u. Gyn. 1898. Bd. 38 S. 465. - Sollen die Lageveränderungen der Gebärmutter operativ be-handelt werden? Ther. d. Gegenw. April 1899. - Dammschutz. Sairan], klin. Vortr. N. F. 1900. No. 278. - Experimentelle Prüfung verschiedener Mtehodßn künstlicher Atmung Neugeborener. Beitr. z. Geb. u. Gyn. (Hegar). 1900. Bd 3. S. 325. - Behandlung des Scheintods Neugeborener* XIII. Congr. intern, de Méd. Sect. d'Obstótr. Paris 1900. - Zur Kenntnis der Todesart des Kindes bei vorzeitiger Lösung der Placenta, Zbl. f. Gyn. 1901. No. 49. -Über die 10 Schwangerschaftsmonate. Zbl. f. Gyn. 1902. No. 2. - Gynäkologie in Irrenhäusern. Mon. f Geb. u. Gyn. 1902. Bd. 15. Ergänzungsheft. S. 383. - Über Dammrisse. Dtsch. Klinik am Eingang des 20. Jahrhunderts. 1902. - Unser Hebammenwesen und die Reform-plane. Samml. klin. Vortr. N. F. 1903. No. 349. - Soil man gleich nach geborenem Kopfe danaoh tasten, ob die Nabelschnur umschlongen ist ? Zbl. f. Gyn. 1903. No. 38. - Die angeblichen Gefahren und die sicheren Vorteile der künstlichen Atmung durch Schw $1 / 8$ gen des tief scheintoten Kindes. Munch, med. Woch. 1905. No. 6. - Tod des Kindes während der Geburt, in Winclcels Handb. d. Geb. 1905. -Die Axendrehung (Cervixtorsion)des myomatösen Uterus. Samml. klin. Vortr. N. F. 1906. No. 410. - Zur Lehre vom Scheintod der Neugeborenen. Ztschr. f. Geb. u. Gyn. 1906. Bd. 57. H. 1. Semmelweißfeier. Corr.-B. d. allg. ärztl. Ver. v. Thür. November 1906. - Spätgeburt. Mon. f. Geb. u. Gyn. 1907. Bd. 25. S. 571. - Lagerung der gebärenden Frau, Lendenbiegsamkeit und aus-treibende Kraft. Beitr. z. Geb. u. Gyn. (Hegar). 1908. Bd. 13. S. 173. - Zur Hebammenfrage. Gyn. Rundsch. 1908. Bd. 2. No. 7. - Über Schwangerschaftsdauer, letzte Regel und Empfängniszeit. Gyn. Rundsch. Bd. 2. No. 10. - Über Querlage, Wendung, Extraktion. Mon. f. Geb. u. Gyn. 1909. Bd. 30. S. 145. - Zur Behandlung des Scheintodes Neugeborener. Ztschr. f. 
Geb. u. Gyn. 1911. Bd. 68. S. 591. - Über die Notwendigkeit gynäkologiseher Diagnose und operativer Therapie in den Irrenhäusern. Gyn. Rundsch. 1912. No. 1. - Zur aseptischen Leitung der Geburten (betrifft Umschlingung der Nabelschnur). Annal. f. d. ges. Hebammenwesen. Bd. 2. H. 4. - Gynäkologie und Psychiatric. Mon.

Hofstätter, Über Befunde etc. 387

f. Geb. u. Gyn. 1914. Bd. 40. S. 276. - Zur Diskussion über Schwanger-schaftsdauer. Zbl. f.

Gyn. 1915. No. 28. - Zur Frage der inneren TJnter-suchung mit einem oder zwei Fingern. Mon. f. Geb. u. Gyn. 1915. Bd. 42. S. 287. - Über den Scheintod Neugeborener und über

Wiederbelebung scheintot geborener Kinder. Samml. klin. Vortr. N. F. 1918. No. 741/42. - Zur Kenntnis von der Einspritzung in die Vene der Nabelschnur bei Blutung irifolge adhacrenter Placenta. Centr. f. Gyn. 1919. No. 15.

F. Skutsch.

Dem Nestor der deutschen Gynäkologie, B, 8,. Schultze, über das Grab hinaus einen wehmutvollen Scheidegruß zuzurufen, ist auch mir Herzensbedürfnis. Ich bin dem geistsprühenden, hochvornehm den-kenden Mann nahegetreten, als er, der Hochgefeierte, dem aufstrebenden, so viel angefeindeten Anfanger mit lebhaftem Interesse entgegenkam. Schultze gehörte zu meinen häufigsten Gästen in der Elsasser Straße, unermüdlich im Verfolgen neuer Gedankengänge, denen er durch seine verständnisvolle Kritik wertvolle Anregungen zu geben nicht ver-schmähte. Seine geistige Frische, sein tiefes Verständnis des Werde-ganges der Frauenheilkunde waren gepaart mit einem so liebenswürdigen Verständnis der heranwachsenden Generation, daß Schulfze wie ein väterlicher Freund unsere Herzen gewann, auch da, wo er es an Kritik nicht feh,len ließ. So wird sein Andenken unter alien, die mit ihm noch in persönliche Berührung kommen durften, fortleben. Die lange Reihe seiner wissenschaftlichen Anregungen wird seinen Namen in der Ent-wicklungsgeschichte der Frauenheilkunde allezeit festhalten A. Martin.

Originalarbeiten.

I.

(Aus dem Instiut îür experimentelle Pathologic in Wien. [VoГstand: Hofrat Prof. Dr. R.

Paltauf. $\uparrow$ )

Über Befunde bei hyperhypophysierten Tieren*).

Von

Dr. B. HOFSTÄTÎER.

1. Einleitung.

Die Erkenntnis der Funktion innersekretorischer Organe ist die Frucht der kritischen Sichtung unendlich zahlreicher einzelner

*) Die Arbeit wurde der Mon. f. Geb. u. Gyn. im September 1917 übergeben; sie kann infolge äußerer TJmstände erst Jetzt abgedruckt werden. Infolge letzterer ist auch das 159 Nummern 
enthaltende Literatur-verzeichnis leider verloren gegangen.

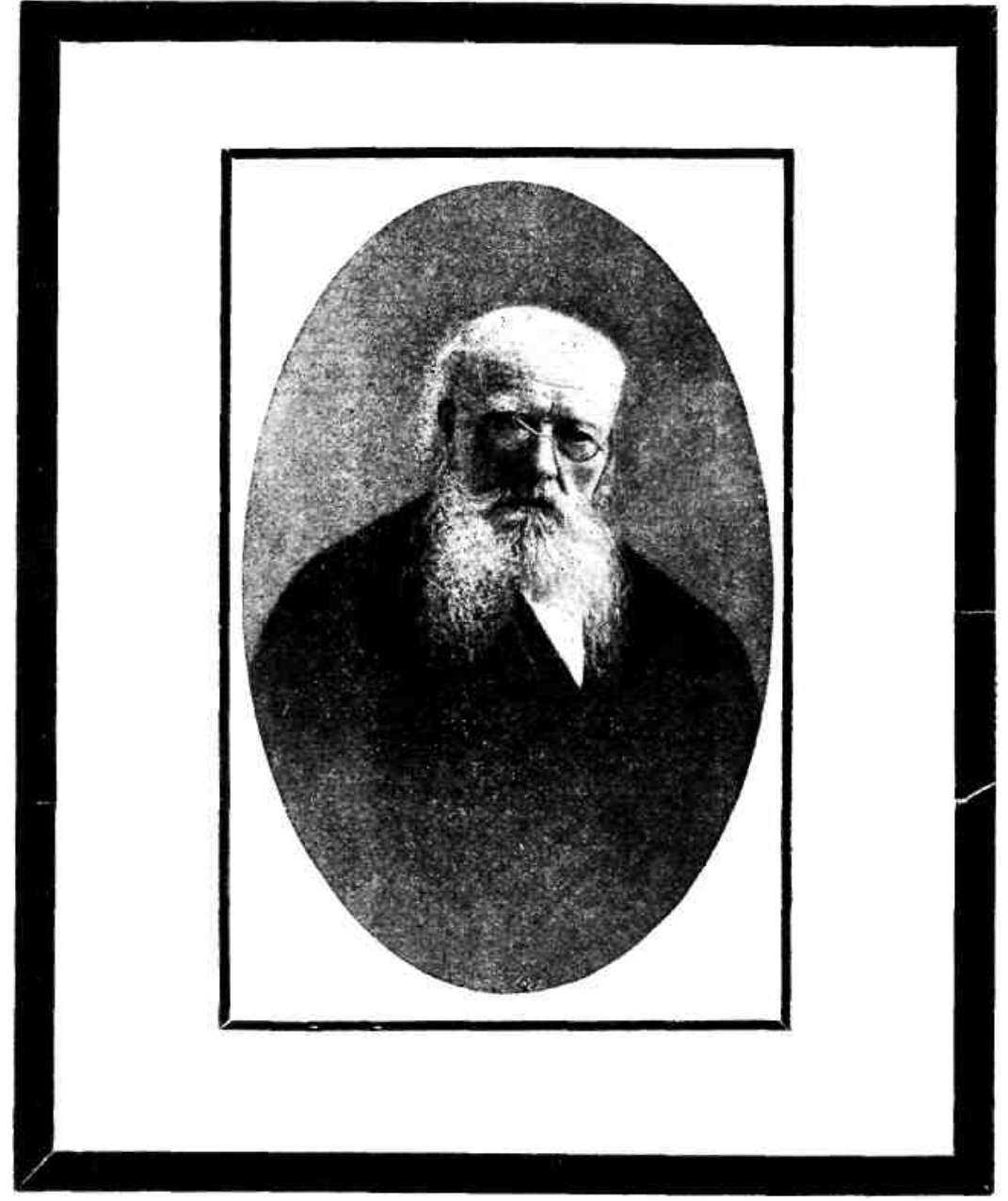

